\title{
Statistics of locally coupled ocean and atmosphere intraseasonal anomalies in Reanalysis and AMIP data
}

\author{
M. Peña, E. Kalnay, and M. Cai \\ Department of Meteorology, University of Maryland, 3431 Computer and Space Science Building, College Park, MD 20742, \\ USA
}

Received: 20 September 2001 - Revised: 2 August 2002 - Accepted: 15 December 2002

\begin{abstract}
We apply a simple dynamical rule to determine the dominant forcing direction in locally coupled oceanatmosphere anomalies in the National Centers for Environmental Prediction/National Center for Atmospheric Research (NCEP/ NCAR) reanalysis data. The rule takes into account the phase relationship between the low-level vorticity anomalies and the Sea Surface Temperature (SST) anomalies. Analysis of the frequency of persistent coupled anomalies for five-day average data shows that, in general, the ocean tends to force the atmosphere in the tropics while the atmosphere tends to force the ocean in the extratropics. The results agree well with those obtained independently using lagged correlations between atmospheric and oceanic variables, suggesting that the dynamical rule is generally valid.

A similar procedure carried out using data from the NCEP global model run with prescribed SST (in which the coupling is one-way, with the ocean always forcing the atmosphere) produces fewer coupled anomalies in the extratropics. They indicate, not surprisingly, an increase in oceandriving anomalies in the model. In addition, and very importantly, there is a strong reduction of persistent atmospheredriving anomalies, indicating that the one-way interaction of the ocean in the model run may provide a spurious negative feedback that damps atmospheric anomalies faster than observed.
\end{abstract}

\section{Introduction}

The coupling of atmospheric flow with slow-evolving anomalous surface boundary conditions, particularly the SST, has the potential to improve the skill of short-term climate prediction (Shukla et al., 2000, and references therein). At the present, two-way coupled atmosphere and ocean models have been used with increasing success in several operational centers to predict the alternating El Niño and La Niña episodes over the tropical Pacific basin as well as the asso-

Correspondence to: M. Peña (mpena@atmos.umd.edu) ciated teleconnection patterns over the extratropics. However, even for El Niño predictions, many coupled models have serious difficulties in producing realistic simulations (e.g. AchutaRao and Sperberg, 2000). The challenge to produce skillful short-term climate predictions from coupled atmosphere-ocean models over the extratropics in the absence of strong tropical SST anomalies still remains. The lack of skillful predictability of the coupled atmosphere and ocean models over the extratropics is partly due to the dominance of synoptic scale atmospheric variability, which is driven primarily by baroclinic instability rather than by lower boundary anomalous forcing. Nevertheless, because of the ocean's larger thermal inertia, the ocean can either strengthen or weaken atmospheric anomalies depending on the phase relationship between ocean and atmosphere anomalies. This in turn depends on whether the coupling is two-way or one-way, and on whether the atmosphere is predominantly forcing the ocean or vice versa.

In the one-way ocean-atmosphere interaction (usually referred to "AMIP runs" for the Atmospheric Model Intercomparison Project), SST anomalies are always assumed to amplify/damp the atmospheric anomalies without any feedback. This approach has been applied in the operational dynamical extended range forecasting (e.g. 15-day daily ensemble forecast at NCEP). The one-way interaction has also been applied in the operational "two-tier" coupled multiseasonal model forecast system in which future SST anomalies obtained from coupled forecasts are used to force an uncoupled atmosphere model to predict the atmospheric anomalies (e.g. Ji et al., 1998). The skill obtained with this approach in the seasonal and interannual predictions is primarily due to relatively skillful prediction of development of El Niño. However, the one-way interaction neglects the feedback effect of the atmosphere on the ocean. This feedback effect could be more important over the extratropics, particularly at the intraseasonal time scale. Moreover, most observational studies indicate that the atmosphere tends to force the ocean over the extratropics at least on intraseasonal time scales (e.g. Palmer and Sun, 1985; Wallace and Jiang, 1987). Therefore, 
this one-way interaction configuration could yield a wrong sign in the coupling fluxes. Masutani (1997) and Hurrell and Trenberth (1999) have revealed the evidence of wrong feedback possibly present in the AMIP runs. They show that in the reanalysis data the correlation between SST anomalies and observed precipitation is positive in the tropics (where the ocean mostly forces the atmosphere), and negative in the extratropics (where the atmosphere mostly forces the ocean), whereas it is positive everywhere in an ensemble of AMIP runs.

There are two prominent local phase relationship patterns observed in Atmospheric General Circulation Model (AGCM) experiments where the atmosphere is forced by a localized midlatitude SST anomaly. The first is a low-level cyclonic vorticity anomaly accompanied with an upper-level anticyclonic vorticity anomaly over a warm SST (e.g. Ting, 1991; Kushnir and Lau, 1992; Kushnir and Held, 1996; Peng and Whitaker, 1999). This configuration can be identified from observations (Mo and Kalnay, 1991). The second is an anticyclonic vorticity anomaly with an equivalent barotropic structure over warm SST anomaly (e.g. Palmer and Sun, 1985; Lau and Nath, 1994; Peng et al., 1995; Peng and Whitaker, 1999).

When the atmosphere drives the ocean, there is more observational consensus about the local phase relationship between quasi-stationary atmospheric and SST anomalies over the extratropics. The observed phase relationship is characterized by a low pressure/cyclonic vorticity anomaly over a negative SST anomaly or a high pressure/anticyclonic vorticity anomaly over a positive SST anomaly (e.g. Mo and Kalnay, 1991; Deser and Timlin, 1997). Figure 1 is a schematic of the consensus configuration that defines the forcing direction, as suggested by Mo and Kalnay (1991). The left panels correspond to anomalies forced by the atmosphere. The dynamical interpretation for the case of no advection (left upper panel) is that when a cyclonic vorticity anomaly occur, the atmosphere rotates faster than the ocean, thus Ekman upwelling and colder temperatures in the ocean are induced. In an opposite situation, when an anticyclonic vorticity occurs, downward radiation and anomalous downwelling are enhanced, thus an increase of sea surface is induced. If advection of colder air from the poles and warmer air from the equator is included (left lower panel), then a cyclonic vorticity anomaly will induce colder SST and shallower lower troposphere west of the center of circulation. The east-west difference in the thickness will result in an eastward tilting. For ocean-driving anomalies (right panels) high SST anomalies induce upward motion and low-level cyclonic vorticity and, when advection is included, a westward tilting occur. This figure also suggests that if we assume that the ocean always drives the atmosphere (as in AMIP runs) the ocean provides a negative feedback on those anomalies where in reality the atmosphere is driving the ocean, and this could result in a faster decay of atmopheric anomalies. This diagnostic rule, if correct, gives a simple but powerful general guidance to distinguish whether the forcing comes from the ocean or from the atmosphere.
In this study of the actual statistics of atmosphere-ocean coupling we make two important simplifying assumption. First, we only consider the local coupling of the atmosphere and the ocean. There are also important remote couplings, such as those resulting in atmospheric teleconnections, but the determination of remote interactions requires a separate methodology, including running coupled systems, that goes beyond this phase of our study. We also neglect the tilting effect of advection shown in the second row of Fig. 1.

Two approaches are compared in this paper: the dynamical rule of Mo and Kalnay (1991) discussed above and lag correlation statistics, briefly described in Sect. 3. We document the frequency of diagnosed ocean-driving versus atmosphere-driving persistent anomalies in the coupled anomalies with time scales ranging from synoptic time-scale to a month, for different seasons, and at different geographical locations. The dynamical technique is applied first to the real coupled ocean-atmosphere system as captured by the reanalysis data (Kalnay et al., 1996; Kistler et al., 2001), and then to a one-way interaction model, AMIP run (Gates et al., 1999).

\section{Data}

This study is based on daily, five-day, and monthly average data from the NCEP/NCAR reanalysis model and on fiveday average data from an NCEP AMIP run. Both models with the same spatial resolution of $2.5^{\circ}$ latitude by $2.5^{\circ}$ longitude. The daily and five-day average data used spans 19 years (1980-1998). The monthly data spans 20 years (19791998) of both reanalysis and NCEP AMIP run data. These three time formats of the reanalysis data allows an assessment of anomalies with time-scales ranging from synoptic to seasonal. In this study we characterize the atmosphere and the ocean conditions with two variables: relative vorticity at $850 \mathrm{hPa}$ and SST.

The zonal and meridional wind components at $850 \mathrm{hPa}$, from which the relative vorticity is obtained, and the SST were obtained via the Climate Diagnostic Center's public web page (http://www.cdc.noaa.gov). The relative vorticity was computed from the wind field using a subroutine from GrADS (Doty, 1995) and a five-day average was then computed from the daily vorticity data. In order to present results uniformly accross the equator, we have deliberately reversed the sign of the relative vorticity field in the Southern Hemisphere. Thus positive vorticity anomalies are cyclonic, and negative anomalies anticyclonic in both hemispheres. The cyclonic vorticity is undefined at the equator.

The SST is the analysis of surface temperature observations using Optimal Interpolation (Reynolds and Smith, 1994). Satellite SST data are obtained from the Advanced Very High Resolution Radiometer (AVHRR) instruments on National Oceanic and Administration (NOAA) polar orbiting satellites, which began operating in November 1981. Prior to November 1981 the Reynolds' SST analyses are reconstructed monthly SST, which were then linearly interpolated 


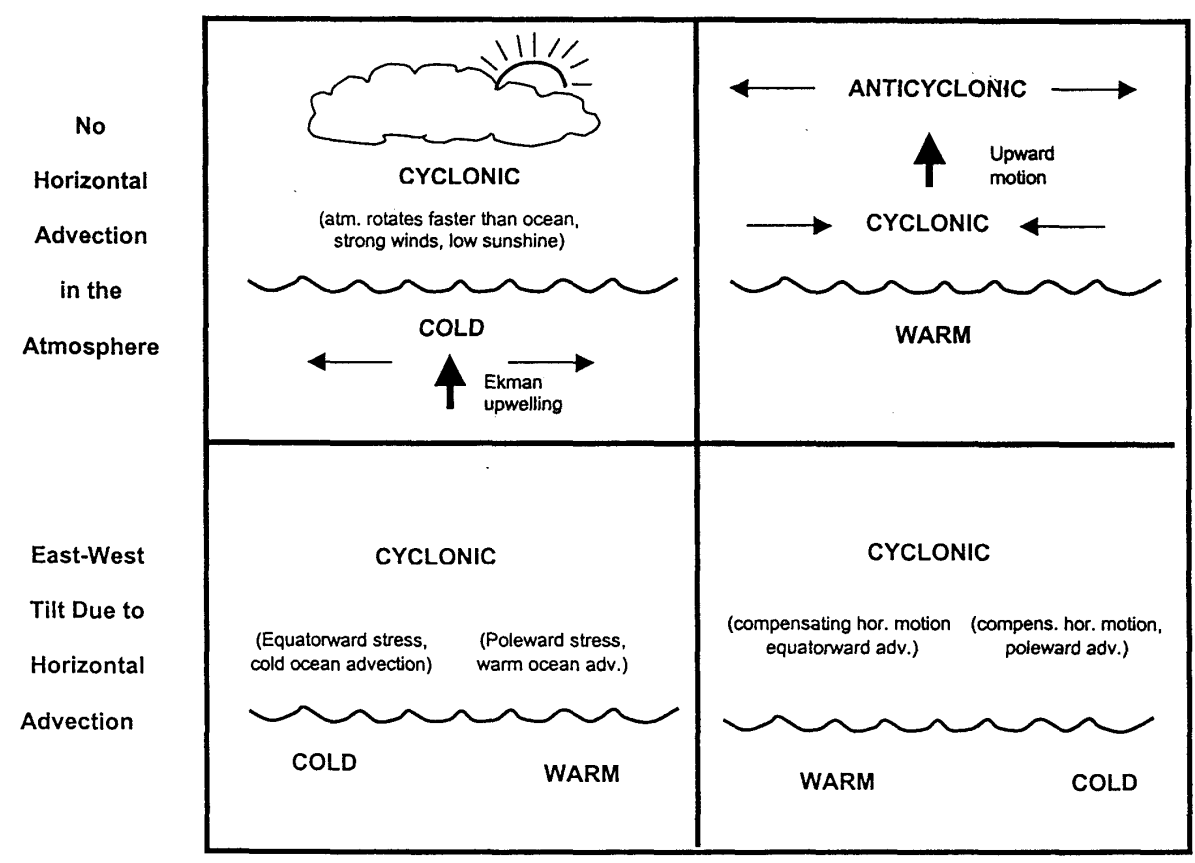

Fig. 1. Schematic of the local phase relationship between SST and low-level atmospheric vorticity depending on whether the atmosphere is driving the ocean (left panel) or the ocean is driving the atmosphere (right panel). The bottom panels indicate the East-West tilt that can be expected from the horizontal advection.

to daily values. The assimilation system in the Reanalysis is updated daily, using a window of 7 days centered on the analysis time. It is important to note that both the atmospheric fields and the SST are obtained from independent assimilations based on observations. Thus the wind field and the SST in the Reanalysis contain (as well as technically possible) the coupling continually generated by nature. The surface fluxes in the Reanalysis are estimated every 6 hours, when observations of wind, air temperature, moisture, etc. are assimilated in the atmospheric model, and reflect their interaction with the observed SST.

To investigate the extent to which a one-way coupling maintains realistic phase relationships daily and monthly $850 \mathrm{hPa}$ wind field data from an NCEP AMIP run for the period 1979-1998 were obtained from NOAA-Climate Prediction Center, CPC, (Jae-Kyung E. Schemm, pers. commun.) and the Program for Climate Model Diagnosis and Intercomparison at the Lawrence Livermore National Laboratory (PCMDI, LLNL), respectively. AMIP runs are atmospheric simulations subject to the observed monthly-average SST and sea ice distribution and standardized values of the solar constant and atmospheric $\mathrm{CO}_{2}$ concentration (Gates et al., 1999). The NCEP AMIP run use the same model (without assimilation of atmospheric observations) and the same resolution as the Reanalysis, which makes the comparison more relevant. However, the results of this comparison should be considered only as a case study since they involve data from only one model.

\section{Methodology}

As indicated in the Introduction, we use both the dynamical rule proposed by Mo and Kalnay to determine the forcing direction in locally coupled anomalies (see Fig. 1) and the traditional lagged time correlation with different lags and leads. In both techniques the annual cycle was subtracted from the time series of SST and relative vorticity. In the daily and five-day average data the leap days were ignored so that all the years in the time series had 365 days. The annual cycle was represented by the first two annual harmonics in the daily and five-day average data, and by the monthly climatology of the 20 years of data in the monthly data.

The dynamical rule is applied first to the reanalysis data and then to the AMIP runs. Low-level vorticity anomalies that are temporally and spatially coincident with the SST anomalies are considered to be "locally coupled". This is because the anomalies considered in both fields are of high amplitude, thus it is very likely that local coupling mechanisms take place to maintain such anomalies. The dynamical rule is applied to all the locally coupled anomalies in the time series. The statistical parameters derived from this technique is the frequency of ocean-driving and atmospheredriving anomalies. To enhance the signal of the statistical parameters, only anomalies with amplitudes of at least one fourth of the standard deviation (local variability) are considered. Because of high variability in the data, standard deviations were smoothed with a sixty-day running mean. To reduce the number of cases of strong fortuitous anomalies that 


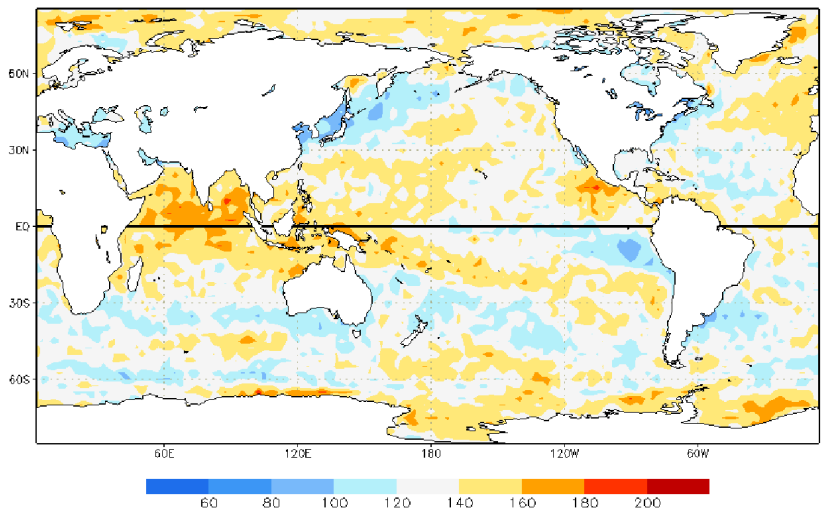

Fig. 2. Number of vorticity anomalies lasting at least 15 days that were simultaneously and locally coupled with SST anomalies. Fiveday average data from the NCEP/NCAR reanalysis. Period: 1 January 1980 - 31 December 1998

arise from errors in the data and may not be representative of the large-scale conditions, only persistent anomalies are considered for each type of time-averaged data. The persistence periods were 15, 25 and 30 days for the 5-day average data, and 2, 3 and 4 months for the monthly data.

We applied the same method to an AMIP run in order to assess how well a one-way coupled model is able to maintain realistic persistent anomalies. We carried out significance tests of the difference in the number of atmospheredriving and ocean-driving anomalies between the Reanalysis and the AMIP run. As an independent technique we computed the lagged correlations between the relative vorticity and the SST anomalies (e.g. Wilks, 1995). This was applied to anomalies with respect to the annual cycle of 5-day and monthly average data.

\section{Statistics of locally coupled anomalies}

The frequency of "ocean-driving" and "atmosphere-driving" anomalies, according to the dynamical rule, is described in this section. For brevity, the discussion will focus in the distribution of 15-day or longer persistent anomalies of the 5-day average data which contains, in essence, the characteristics of sub-seasonal locally coupled anomalies. Figure 2 shows the distribution of the number of locally coupled persistent anomalies of the vorticity field from the reanalysis data. We see a minimum number in the El Niño region because the anomalies are much longer lasting due to the coupling between the ocean and the atmosphere. On the other hand in the midlatitude storm tracks regions we also see a minimum reflecting the transient characteristics of the circulation. Regions of relatively large number of locally coupled anomalies include the eastern tropical Pacific of the Northern Hemiphere, the Indian Ocean and the warm pool region.

We now apply the dynamical rule to these simultaneous, locally coupled anomalies. The percentage frequency distribution of ocean-driving and atmosphere-driving anomalies

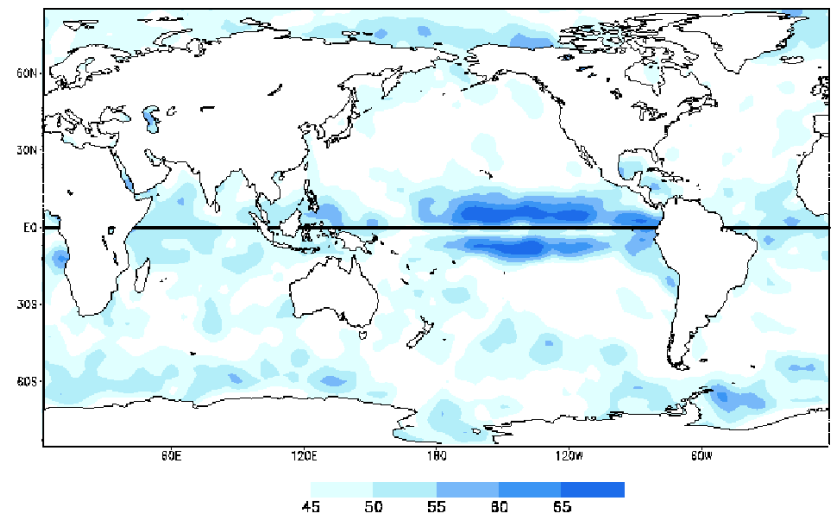

Fig. 3. Percentage of the observed coupled anomalies lasting more than 15 days that are "ocean-driving". Data from the NCEP/NCAR reanalysis. Period: 1 January 1980-31 December 1998

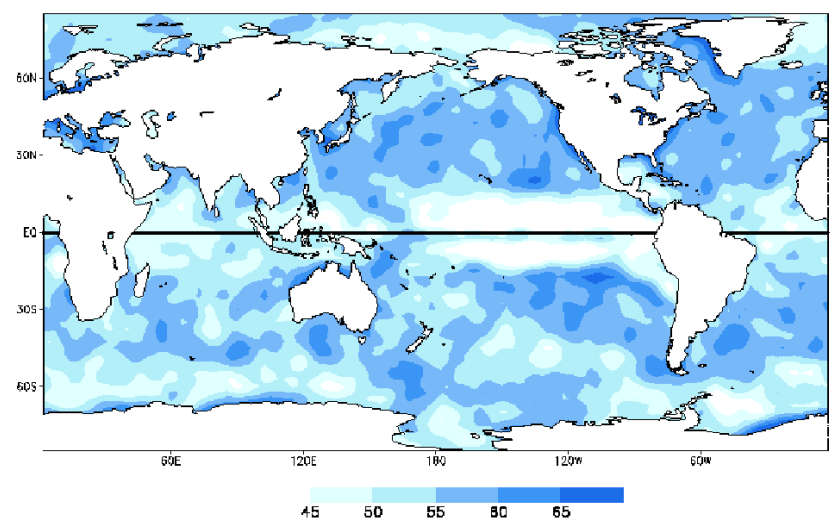

Fig. 4. Percentage of the observed coupled anomalies lasting more than 15 days that are "atmosphere-driving". Data from the NCEP/NCAR reanalysis. Period: 1 January 1980 - 31 December 1998.

persisting at least 15 days is shown in Figs. 3 and 4, respectively. The distribution of ocean-driving anomalies (Fig. 3) shows a maximum in the central equatorial Pacific decreasing in the midlatitude region of both hemispheres, indicating a tendency of more "ocean driving the atmosphere" in the tropics. On the other hand, Fig. 4 indicates a tendency for "atmosphere driving the ocean" throughout most of the extratropics, particularly in regions of the subtropical anticyclones. The tendency of ocean driving the atmosphere in the tropics and atmosphere driving the ocean in the extratropics is also observed by applying the dynamical rule to the monthly and the daily persistent anomalies (not shown).

\section{Comparison with an AMIP run}

Since intraseasonal predictions are usually done with oneway interaction models, in which the atmosphere does not influence the ocean SST, as in the AMIP run, it is important to assess how closely these models represent the statistics observed in the reanalysis. We will also refer to atmospheric persistent anomalies in the AMIP run that occur simultane- 


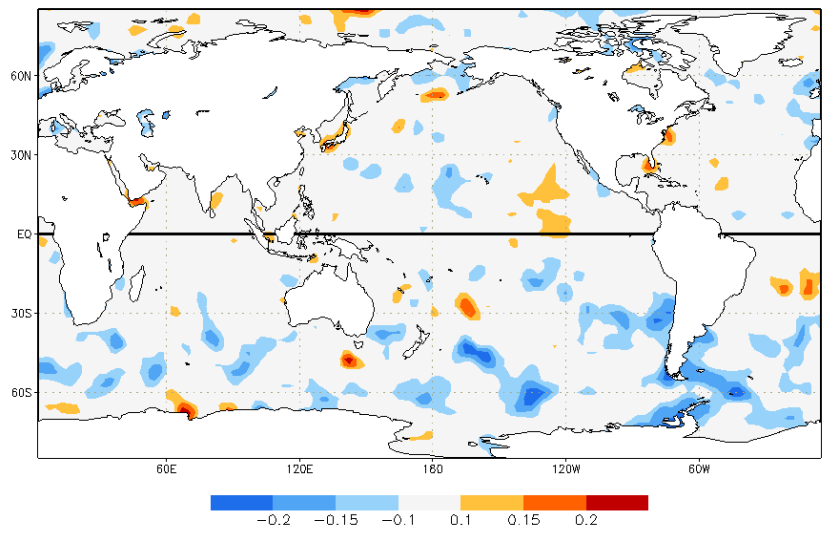

Fig. 5. Relative difference in the frequency of persistent coupled anomalies between the AMIP run and the reanalysis [(AMIPNCEP)/AMIP].

ously with SST anomalies as "locally coupled anomalies". Figure 5 shows the difference in the number of cases of coupled anomalies in the AMIP run minus the reanalysis (given in Fig. 2) divided by the total number of anomalies. The relative differences are within $\pm 10 \%$ in most of the tropical oceanic region and in the Northern Hemisphere; however, there is a clear bias towards fewer coupled persistent anomalies in the AMIP run than in the reanalysis, especially in the extratropics. Fewer number of persistent anomalies in the AMIP run suggests that in one-way interaction models, in which atmospheric feedback is ignored, the prescribed ocean SST will tend to provide a spurious negative feedback and therefore damp the atmospheric anomalies, as suggested by the schematic of Fig. 1.

The difference in the number of "ocean-driving" anomalies between the AMIP run and the reanalysis and its statistical significance is presented in Fig. 6. In red are the regions where the number of "ocean-driving" anomalies in the AMIP run exceeds the number in the reanalysis at a $95 \%$ confidence limit, which occurs in most of the extratropics. There are some areas with more ocean-driving anomalies in the reanalysis than in AMIP, but they are confined to small regions in the tropics and are not statistically significant. A larger number of "ocean-driving" anomalies over most of the extratropics is not surprising, given that in the AMIP run the atmosphere does not influence the ocean. Conversely, there are fewer anomalies in the extratropics with "atmospheredriving" phase relationship in the AMIP run than in the reanalysis (Fig. 7). This decrease in long-lasting "atmospheredriving" anomalies in the AMIP run overcompensates for the increase in "ocean-driving" anomalies. The net result is the reduction in the total number of anomalies in the extratropics shown in Fig. 5.

\section{Lag correlation analysis}

We now compare the results obtained with the dynamical rule with those derived from lag correlations. Figure 8a shows

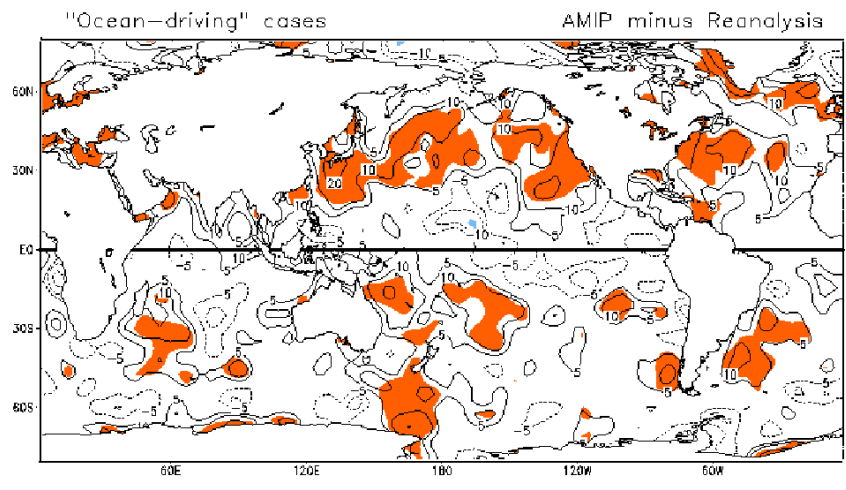

Fig. 6. Difference of "Ocean-driving" anomaly cases between the AMIP run and the Reanalysis, and confidence limits of $95 \%$ to reject the null hypothesis that the number of "ocean-driving" anomalies is the same. The alternative hypothesis is that the number of "ocean-driving" anomalies is larger in the AMIP run (red).

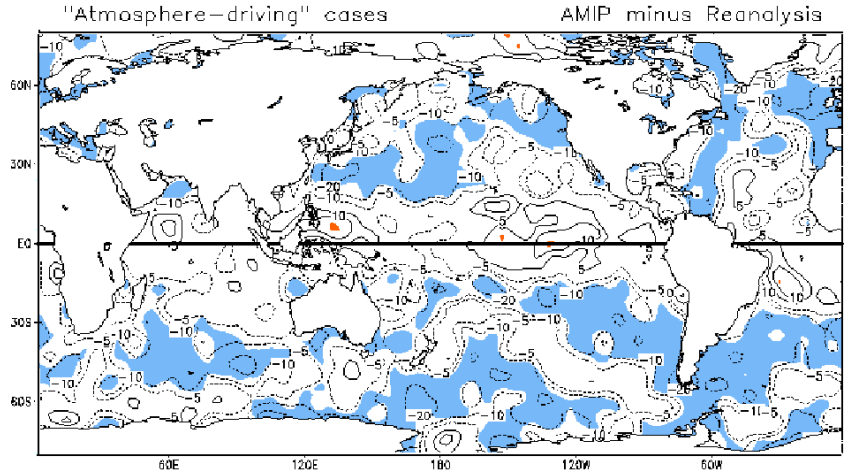

Fig. 7. Difference of "Atmosphere-driving" anomaly cases between the AMIP and the Reanalysis, and confidence limit of $95 \%$ to reject the null hypothesis that the number of "atmosphere-driving" anomalies is the same. The alternative hypothesis is that the number of "atmosphere-driving" anomalies is larger in the Reanalysis (blue).

the simultaneous cross-correlations between the $850 \mathrm{hPa}$ cyclonic vorticity and the skin temperature of the monthly reanalysis data. The correlation is positive in the tropics (i.e. cyclonic over warm) and negative in the extratropics (i.e. cyclonic over cold) in good agreement with the dynamical rule. The change in sign of the correlation between the tropics and the extratropics in the reanalysis is also consistent with the correlation pattern found between SST and precipitation (Hurrell and Trenberth, 1999). It is apparent that the central equatorial Pacific region with the highest frequency of ocean-driving anomalies (Fig. 3) agrees with the largest positive correlation. In the 1-month ocean-leading correlation (Fig. 8b), the tropics remain with positive correlation, indicating that the ocean-driving anomalies are long lasting. In the extratropics, on the other hand, the correlations become much smaller than the simultaneous correlations, indicating the absence of long-lasting ocean-driving anomalies. By contrast, in the 1-month atmosphere-leading correlation (Fig. 8c left), there is a stronger negative correlation than in 
NCEP/NCAR reanalysis
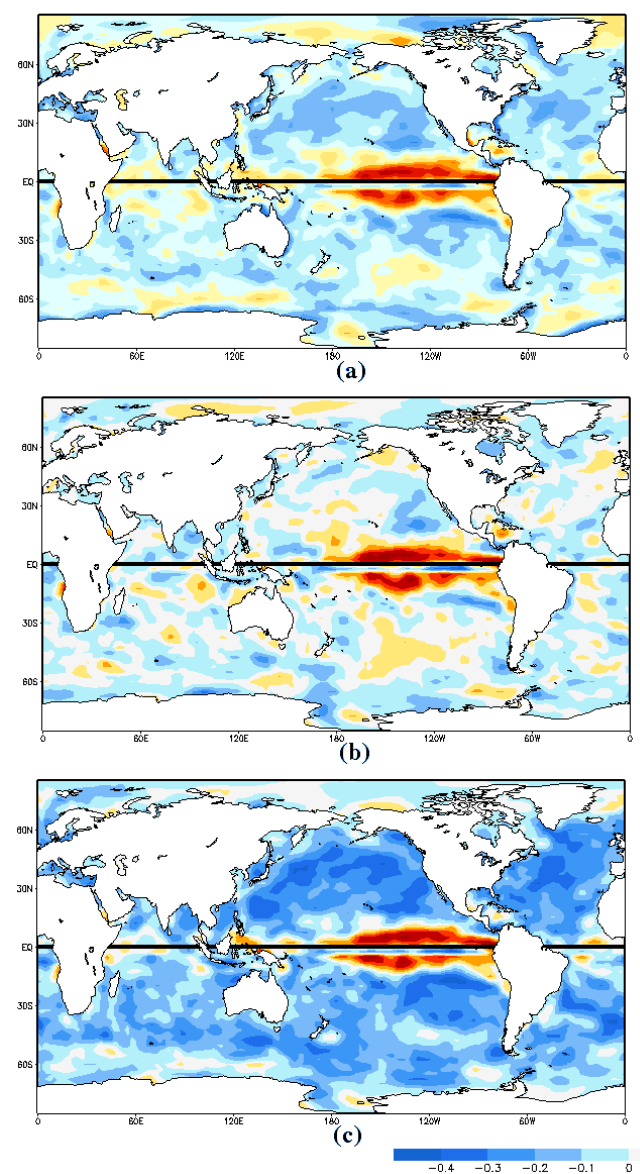

NCEP AMIP run
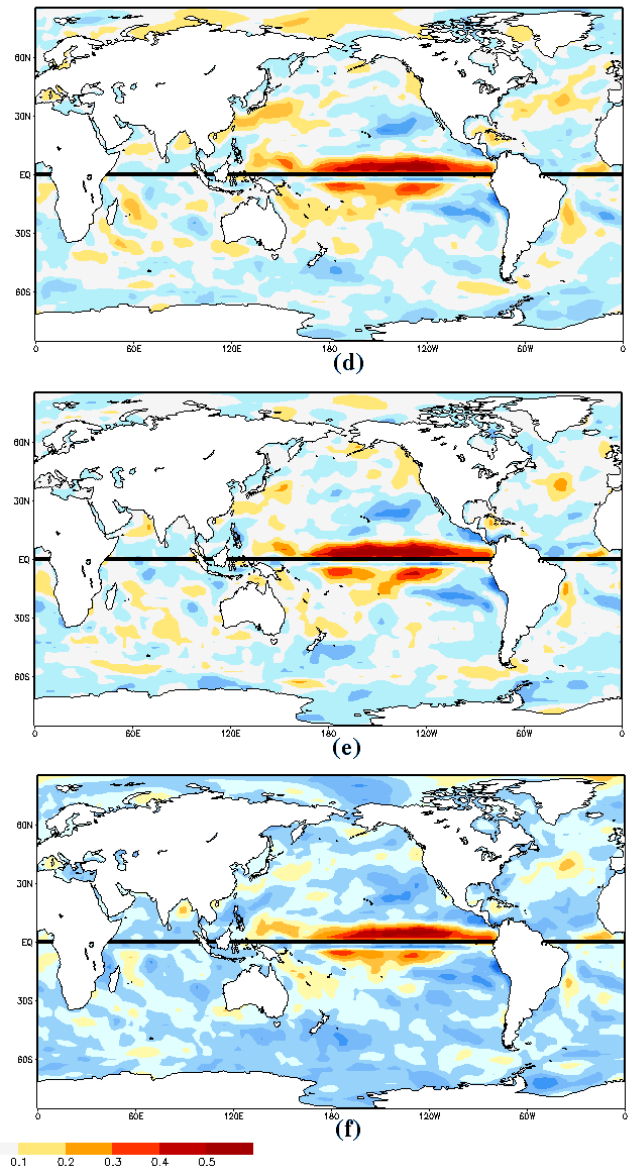

Fig. 8. (a, d) Simultaneous (b, e) One-month SST leading and (c, f) One-month vorticity leading correlation function in the monthly NCEP/NCAR reanalysis data (left) and the AMIP run (right). Period: January 1979 - December 1998

the simultaneous correlation in the extratropics, indicating not only that the atmosphere tends to preceed the ocean but also that there are long-lasting atmosphere-driving anomalies. Over the tropics, especially in the central and eastern Pacific, the correlation does not change much with the lag/lead, due to the fact that the El Niño anomalies are very long-lasting. Similar differences in the lag and lead correlations are suggested, but not as clearly, in the five-day and daily data (not shown).

The simultaneous correlation of the monthly vorticity from the AMIP run with the SST (Fig. 8d) indicates also a large positive correlation in the deep tropics and generally negative in the extratropics. However, the region of negative correlation is smaller than in the reanalysis, indicating a tendency of more "ocean-driving", according to the dynamical rule. There are several areas where the sign of the correlation is opposite in the AMIP and in the reanalysis (e.g. the warm pool, the Indian Ocean, and the North Atlantic). Little difference is observed in the 1-month ocean leading (Fig. 8e) with respect to the simultaneous correlation in the AMIP run data. The 1-month atmosphere leading (Figs. 8c and f), on the other hand, shows a dramatic difference with higher correlation in the reanalysis than in the AMIP run. Once again, these correlation results agree well with the dynamic rule.

\section{Summary and discussion}

In this paper a climatology of persistent locally coupled anomalies was developed according to the main forcing direction in the interaction of the ocean and atmosphere. A simple dynamical rule that considers the local phase relationship between the SST and the low-level vorticity is applied first to the NCEP reanalysis data and then to an AMIP run. The results are consistent with previous observational studies (e.g. Davis, 1976; Palmer and Sun, 1985; Wallace and Jiang, 1987; Deser and Timlin, 1997), which suggest that, in general, atmospheric anomalies in the tropics tend to be forced primarily by the ocean, whereas in the extratropics ocean anomalies tend to be forced by the atmosphere. The results using two independent techniques, the lag correlation statistics and the phase relationship of the SST and the vorticity agree well with each other and confirm the tendency of ocean-driving in the tropics and atmosphere-driving in the extratropics. It should be noted the dynamical technique considers the anomalies on a case-by-case basis, which allows 
further flexibility in the classification and compositing analysis with other variables of the ocean-atmosphere system.

For the 15 days or longer persistent anomalies in the 5-day average data, the AMIP simulation contains a number of the persistent coupled anomalies similar to the reanalysis data, particularly for "ocean-driving" anomaly cases. The differences occur mostly in the atmosphere-driving cases, where the AMIP run present much fewer cases. This agrees well with the notion that in AMIP runs with one-way interaction of the ocean, there is an erroneous negative feedback from the ocean with the result that atmospheric anomalies are damped faster than in reality.

Our results suggest that assuming an ocean-driving scenario to couple the atmosphere with anomalous lower boundary conditions may not be optimal to produce skillful predictions. This study identifies the regions when the atmosphere feedback is not negligible. Further questions that remain to be addressed include the effect of the horizontal advection, and the extent to which remote forcing plays a role to generate or maintain the locally coupled anomalies considered in this study. We have only considered either atmospheredriving or ocean-driving anomalies, i.e. primarily one-way interactions; however, there must be obviously situations in which two-way interactions are important. The analysis of complex two-way interactions and of remote coupling requires experimentation with coupled models.

Acknowledgements. This research was supported by the NOAAOffice of Global Programs. The authors thank Jae-Kyung E. Schemm (NOAA-CPC) for kindly providing the daily AMIP run dataand to two anonymous reviewers whose suggestions greatly improved the paper.

\section{References}

AchutaRao, K. and Sperberg, K.: ENSO in coupled GCMs, PCMDI report no. 61, Tech. rep., Program for Climate Model Diagnostics and Intercomparison, 2000.

Davis, R.: Predictability of sea surface temperature and sea level pressure anomalies over the North Pacific Ocean, J. Phys. Ocean, 6, 249-266, 1976.

Deser, C. and Timlin, M.: Atmosphere-Ocean interaction on the weekly timescales in the North Atlantic and Pacific, J. Climate, 10, 393-408, 1997.

Doty, B.: Grid Analysis and Display System. Freeware available at http://dao.gsfc.nasa.gov/software/grads, 1995.

Gates, L., Boyle, J. C., and Dease, C. C.: An Overview of the Results of the Atmospheric Model Intercomparison Project (AMIP I), Bull. Amer. Meteor. Soc., 80, 29-55, 1999.

Hurrell, J. and Trenberth, K.: Global SST analyses: Multiple problems and their implications for climate analysis, modeling and reanalysis, Bull. Amer. Meteor. Soc., 80, 2661-2678, 1999.

Ji, M., Behringer, D., and Leetmaa, A.: An improved coupled model for ENSO prediction and implications for ocean initialization. part ii: The coupled model, Mon. Wea. Rev., 126, 1022-1034, 1998.

Kalnay, E., Kanamitsu, M., Collins, W., and Saha, S.: The NCEP/NCAR 40-year reanalysis project, Bull. Amer. Meteor. Soc., 77, 437-471, 1996.

Kistler, R., Kalnay, E., Collins, W., and Saha, S.: The NCEP/NCAR 50-year reanalysis: Monthly means CD-ROM and documentation, Bull. Amer. Meteor. Soc., 82, 247-268, 2001.

Kushnir, Y. and Held, I.: Equilibrium atmospheric response to north atlantic SST anomalies, J. Climate, 9, 1208-1220, 1996.

Kushnir, Y. and Lau, N.-C.: The general circulation model response to a North Pacific SST anomaly: Dependence on time scale pattern polarity, J. Climate, 5, 271-283, 1992.

Lau, N.-C. and Nath, M.: A modeling study of the relative roles of tropics and extratropical SST anomalies in the variability of the global atmosphere-ocean system, J. Climate, 7, 1184-1207, 1994.

Masutani, M.: Relation between SST and rainfall on the seasonal time scale, in Proc. 22nd. CDP Workshop, edited by AMS, pp. 65-68, American Meteorological Society, 1997.

Mo, K. and Kalnay, E.: Impact of the sea surface temperature anomalies on the skill of monthly forecasts, Mon. Wea. Rev., 119, 2771-2793, 1991

Palmer, T. and Sun, Z.: A modeling and obervational study of the relationship between sea surface temperature in the north-west Atlantic and atmospheric general circulation, J. Roy. Meteor. Soc., 111, 947-975, 1985.

Peng, S. and Whitaker, J.: Mechanisms determining the atmospheric response to midlatitude SST anomalies, J. Climate, 12, 1393-1408, 1999.

Peng, S., Mysak, L., Ritchie, H., Demore, J., and Dugas, B.: The differences between early and midwinter atmospheric responses to sea surface temperature anomalies in the northwest Atlantic, J. Climate, 8, 137-157, 1995.

Reynolds, R. and Smith, T. M.: Improved global sea surface temperature analyses using optimum interpolation, J. Climate, 7, 929948, 1994

Shukla, J., Anderson, J., Baumhefner, D., Brankovic, C., Chang, Y., Kalnay, E., Marx, L., Palmer, T., Paolino, D., Ploshay, J., Schubert, S., Straus, D., Suarez, M., and Tribbia, J.: Dynamical Seasonal Prediction, Bull. Amer. Meteor. Soc., 81, 2593-2606, 2000.

Ting, M.: The stationary wave response to a midlatitude SST anomaly in an idealized GCM, J. Atmos. Sci., 50, 2845-2867, 1991.

Wallace, J. and Jiang, Q.: On the observed structure of the interannual variability of the atmosphere/ocean climate system, in Atmospheric and Ocean Varibility, (Ed) F. Cattle, pp. 17-43, Royal Meteorology Society, 1987.

Wilks, D.: Statitical Methods in the Atmospheric Sciences, Academic Press, 1995. 